his pulse was much excited, and he had a now opened a vein in the arm, and algood deal of fever. He stated that eighteen lowed the blood to flow until syncope suyears ago he was under the care of Sir pervened, which did not take place until EVEfa RD HoMe for strictures of the thirty ounces of blood were abstracted. urethra, then having three strictures, each In two hours I visited her again, when I of which was destroyed by caustic. He had the satisfaction to find, that the urine added that he returned to India in perfect had been escaping in small quantities. Its health, introducing a bougie occasionally flow gradually increased, and the bladder for some years, but that no bongie had passed for the last ten years, and that during the last few month he had had several attacks of retention, the stream of urine having been for a considerable time preternaturally small. He had used no remedies except during the periods of retention, on which occasions no instruments could be passed into the bladder, the attacks yielding to large doses of opium, and repeated local and general bleedings. $\mathrm{He}$ enjoyed perfect general health, and had been in India forty-fire years.

After placing him in bed, I opened a vein in his arm, and abstracted upwards of forty ounces of blood before the pulse sank. He had already taken sixty drops of laudanum, and sixty more were now given. Two hours after, the urine began to escape. He then took six grains of calomel, and passed a better night than usual. On the following day the size of the stream was larger than it had been for some time, and the irritation of the urethra was so much diminished that he was called on to make water only once during the next night. Notwithstanding the depletion, and the rigid antiphlogistic regimen which was pursued for some days, he experienced no diminution of his muscular strength. The effects of this attack being in a few days subdued, I succeeded, after numerous careful attempts, in introducing the smallest-sized elastic-gum catheter into the bladder, where it was retained, and a succession of instruments of larger and larger sizes were afterwards introduced, so that in a few weeks the contracted urethra was dilated, and now, after the lapse of several years, I have learned that he continues well.

Case 4.-A married lady, upwards of thirty years of age, after having suffered much pain and irritation in the parts around the external orifice of the vagina, was attacked with retention of urine. I found the bladder very much distended, accompanied by a great deal of febrile excitement. A catheter passed readily along the urethra till it reached near the bladder, where it met with a firm resistarice. Instead of using any force, I withdrew the catheter, conceiving that the inflamed state of the parts and the spasmodic contraction of the urethra had caused the obstruction. copiously evacuated, and in a few days her health was restored.

Remarks.-These cases will, I hope, suffice to point out the practical importance of duly deliberating on such occa. sions, before adopting or persisting in the use of the catheter or knife as the means of relief. They teach us the propriety of endeavouring to discover the cause of the retention of urine, before employing any mechanical means for evacuating the bladder. Whatever may be the precise nature or extent of disease in the urinary passages, when an accidental retention takes place, the inability to void the urine must arise only from some temporary cause, and therefore it ought to be the first duty of the surgeon to trace what that cause is, and endeavour to remove it. In most instances the retention will be found to be the effect of a temporary inflammation, which inflammation is usually brought on by the abuse of spirituous liquors,-by some over-excitement of the organs, - or by some disturbed state of the system generally.

CASE OF

\title{
TUMOUR OF THE ABDOMEN
}

CONNTCTED WITH REMARKABLE

\section{DISEASE OF THE LUNGS.}

By William Tomkins, Esq., M.R.C.S. $\& ; c$, Yenvil, Somerset.

Case-I was applied to by Charles Parker, æatat. 28, of delicate make and strumous habit, on account of a protrusion from the abdomen, which he conceived was a ventral hernia. He informed me that he discerned the tumour for the first time about two months previously, after violent walking exercise. It was then small, and slightly painful, but it had gradually increased, and now appeared about the size of a hen's egg, without the slightest dis. coloration or tenderness of the skin. On examining the swelling, it presented to the eye the precise appearance of a ven. tral hernia. It protruded midway between the umbilicus and the spine of the ileum on the right side. I laid the patient in a 
recumbent posture and applied pressure, | and faintness. I applied such medical on which the swelling instantly receded treatment from day to day as I thought with a slight crackling noise; but the man the circumstances of the patient required, complained of faintness, palpitations, a and which it would be tedious to detail. sense of suffocation, and tickling in the In a short time the abdominal swelling throat, which I could not exactly account gave way at the lower cicatrix left by one for, as he did not appear to have any ti- of the setons; the cough directly became midity about him. I desired him to stand up and cough, and on his doing so, the swelling reappeared immediately.

As I was not satisfied respecting the nature of the complaint, I desired the patient to call on me again in about ten days, which he did. I then found that the tumour was nearly double its original size, but still capable of being returned easily on applying pressure in the recumbent position. The general health of the patient was tolerably good; indeed he complained of nothing but slight dyspnoea on walking against hill. I now felt satisfied that the swelling contained a fluid, and, therefore, determined to make an opening with a couching needle, in the manner recommended by the late Mr. Hey in cases where there was doubt. I did so, and on withdrawing the needle a "thin serous fluid" escaped. I then dilated the wound with a bistoury, and let out about twelve ounces. I applied a dressing of adhesive plaster, and pressure, and the wound healed by adhesion in a few days.

About a month elapsed before the man came to me again, when $I$ found the swelling larger and more diffused. I opened it and let out a pint of fluid similar to that before evacuated. On examining the cyst with a probe I could not trace any communication between it and the abdo. minal cavity, or any sinus beyond its apparent parietes. I therefore determined to pass two setons, one from below upwards and another across, and in a few days there was a good purulent discharge, which gradually lessened, and after a month $I$ was enabled to remove the setons, and had the satisfaction to see the wound perfectly healed and the cyst adherent.

I lost sight of the patient for nearly six months; he had returned to his work, and felt but little inconvenience from his com. plaint ; now, however, he complained of cough and dyspnoe, which he said had come on gradually for about ten days, and a return of the tumour, accompanied by much more pain than he had before felt. He had quick pulse, slight expectorations, and night perspirations. There was little sourd in the right side of the thorax on percussion. Coughing produced a swelling in the site of the original tumour, which, when returned by means of pres. sure in the recumbent posture, excited cough, dysproea, palpitation, quick pulse,

No. 573 . more troublesome, and whenever it occurred, a volume of air rushed through the wound, which I found it impossible to prevent by any means that I could devise. I tried oiled silk corered by adhesive plasters, bandages, \&c.

My attention was now called to a new feature of the case. The man complained of a most acute pain, together with a swelling by the side of the breast bone, which passed in and out, as he breathed, with a crackling noise. On examination, 1 found a protrusion of the lung under the skin, which could be distinctly felt to crepitate between the fingers. It was about the size of a hen's egg, and passed out just under the second rib on the right side, close to its sternal articulation. I tried to restrain this pulmonary hernia, if I may so term it, by means of pressure, but the man could not long endure it, and would not keep anything over the part. The general health now declined, and it was evident that the right lung was much diseased. The cough and expectoration were corsiderable, as well as the discharge and escape of air from the abdominal ulcer, which had now extended itself to the extent of several inches, and assumed a very unhealthy appearance.

I need not particularize the progress of the case from time to time; suffice it to say, that the pulmonary symptoms gradually increased for about three months, when the rupture of the lung, which had considerably enlarged and had adhered to and inflamed the skin for a fortnight, gave way, and an external opening was found, through which a purulent discharge passed. This opening gradually extended itself from day to day, and on the fifth day from its giving way, the patient respired and expired almost as freely through the wounds in the abdomen and thorax, as he did by the trachea. It was impossible to prevent this, though I tried every means, as the rush of air and the discharge from the lungs removed everything I could apply externally. Several of my professional friends saw the case with me, and it is astonishing that the patient lived upwards of three weeks in this situation. Mr. Latham, my assistant, and myself, opened the body in the presence of Dr. Penkivil, an intelligent and respectable physician of this town, and the following were the 


\section{Post-mortem appearances.}

The right lung was completely consolidated, and united to the thorax by inseparable adhesions. The sternal end of the second rib was absorbed, where the portion of lung forming the hernia had protruded. There was a large cyst in the centre of the right lung, empty, but of sufficient size to contain four ounces of pus. From the lower part of this cyst ran a sinus, which passed down behind the diaphragm as low as the eighth rib, an inch of which was completely absorbed, and a separated portion of half an inch was lying loose; at this place the matter had made its way ont, and there ran down under the skin to the spot where the tumour was first observed. The heart was of its usual size, but the pericardium was thickened, and contained two ounces or nore of pure pus. The abdominal viscera were healthy, as was the left lung.

Remarks.-The perusal of this case must present to the reader many striking and remarkable features. It is in the first place very extraordinary that the abscess of the lung should have proceeded so far, and even have pointed in the abdomen, when, excepting the dyspncea, there was not the slightest indication of pulmonary disease; but cases of extensive and even fatal disease of the lungs do sometimes occur in very strumous habits, and proceed almost to their termination before the urgency of the symptoms induces the patient to apply for professional aid; indeed such instances have frequently come under my notice. The great similarity of the swelling in the first instance to a ventral hernia, and the crepitating feel, produced most probably by an escape from the aircells, are well worthy of remark, and show us the importance of an attentive and well-considered examination in every case of tumour, before venturing on an operation. Again, the healing of the cyst and the non-accurnulation of any fluid in the part for several months, is a fact to me quite inexplicable, as it was evident from the post-mortem examination that neither the cyst in the lung or the sinus leading from it could have closed; and yet as the patient was for months free from cough or expectoration, we cannot imagine (coupling these circumstances with the lower cyst remaining impervious) that any accumulation could have taken place in the lung, or how comes it that it did not gravitate? Are we then to infer that such cysts do secrete? I presume so, and if we admit the fact, what suspended its action? It is a most curious circumstance that a portion of the lung should have prom truded after the cyst had a free vent by a dependent opening, and it is more singular that the portion of lung which protruded felt perfectly soft and healthy for at least seven or eight weeks after it was first discovered. There would have been nothing remarkable if purulent matter had pressed on the rib from an abscess of the lung and caused its absorption; but for a healthy portion of the lung to effect this, is to me a circumstance of a very unaccountable nature. The length of time the patient lived after the two external openings of communication were formed with the lungs, is another proof of the extraordinary powers which nature frequently exhibits to us in her modes of accommodation to the influence of diseased actions and altered structures. The quantity of purulent matter in the pericardium was a circumstance of very rare occurrence, and it is particularly worthy of notice that dur. ing life there were no marked symptoms of such disorder. 1 do not recollect ever to have felt the slightest intermission of the pulse, except when the jalpitations and faintness occurred from my making pressure on the tumour in the abdomen.

Having said thus much, I leave this (I presume unique) case to the reflections and observations of the numerous, intelligent, and respectable readers of that excellent and straightforward publication, The LaNCET.

Yeovil, Somerset, August 12th, 1834.

ON THE TREATMENT OF

\section{MALIGNANT CHOLERA,}

CHIEFLY WITH

\section{EMETICS OF COMMON SALT.}

\section{By George Beaman, Esq., M. R. C. S, London.}

During the last three months I have attended several cases of cholera, and the following plan of treatment has been so extremely successful that I am induced to trouble the Editor of The LANCET with this communication, hoping it may benefit some of my professional brethren.

The syinptoms of cholera in its worst form are too well known to require description, and to this stage of the disease, I wish it to he understood, the following plar of treatment is especially applicalle.

To an adult I give three tablespoonfuls of common table salt in half a pint of tepid or cold water; this produces romiting in less than one minute, and the vomiting ought to be powerfully violent; such effect is followed by an immediate 